\title{
Regularity and Chaos in the Hydrogen Atom Highly Excited with a Strong Magnetic Field
}

\author{
M. Amdouni ${ }^{1}$ and H. Eleuch ${ }^{2}$ \\ ${ }^{1}$ Bizerte Preparatory Engineering Institute, University of Carthage, 7021 Zarzouna, Tunisia \\ ${ }^{2}$ Department of Physics, McGill University, Montreal, QC, Canada H3A 2T8
}

Correspondence should be addressed to H. Eleuch; heleuch@physics.mcgill.ca

Received 19 August 2014; Revised 17 November 2014; Accepted 17 November 2014; Published 3 December 2014

Academic Editor: Yan-Jun Liu

Copyright (C) 2014 M. Amdouni and H. Eleuch. This is an open access article distributed under the Creative Commons Attribution License, which permits unrestricted use, distribution, and reproduction in any medium, provided the original work is properly cited.

The effects of the relativistic corrections on the energy spectra are analyzed. Effective simulations based on manipulations of operators in the Sturmian basis are developed. Discrete and continuous energy spectra of a hydrogen atom with realistic nucleus mass in a strong magnetic field are computed. The transition from regularity to chaos in diamagnetic problem with the effect of the nucleus recoil energy is explored. Anticrossing of energy levels is observed for strong magnetic field.

\section{Introduction}

A lot of works have been recently dedicated to the study of two- or multilevel systems such as Rydberg atom, excitonic systems, and nanoresonators [1-13]. Its great importance lies in its implication in different branches of physics: astrophysics [9, 12], atomic physics [14-17], molecular physics [18, $19]$, spectroscopy [20, 21], solid state [22], and plasma physics [23]. It leads to interesting quantum features such as soliton propagation [24-30], entanglement [31,32], antibunching [33], squeezing [34], bistability [35], and chaos [36, 37].

The Rydberg atom in a static magnetic field is a simple system which can be investigated experimentally and theoretically. It turns out a prototype of quantum systems whose classical behavior may show chaotical dynamics [38-42]. Chaotic appearance results in the fact that for certain initial conditions the behavior of the dynamical system becomes unpredictable $[43,44]$. Chaos is identified by high sensitivity to the initial conditions. Indeed a system with $n$ degrees of freedom is regular if there are $n$ independent constants of motion [45]. These constants of motion allow remaining unchanged, for instance, the regularly spaced energy levels [38].

Consequently many researches have been carried out to discover the connections between classical and quantum chaos through Rydberg atoms in strong static fields [46, 47].
To a large degree, the justification for this concern is attributable to the relevance of chaos in manifold branches of science such as physical, chemical, biological, electrical, and socioeconomical systems [38, 40, 41, 48-54]. In physics, this problem is related to the atomic magnetism [55] especially to explore the impact of the diamagnetic effect $[38,56]$ on the energy diagrams: the overlapping and the anticrossing of energy levels. Several techniques [57, 58] have been performed to explore the complete part of a magnetic field.

The hydrogen atom in a strong magnetic field was explored $[3,12,38,55,59,60]$. The hydrogenoid and nonhydrogenoid atoms in the presence of the external field were also studied $[38,59,61,62]$. In the regime of strong external field, Delande has shown that the system behavior is qualitatively similar to that of the hydrogen atom [59]. All these works were limited to the nonrelativistic case. In our previous paper [63], we have studied the effect of relativistic corrections on the regularity behavior of the hydrogen atom in a strong static magnetic field. This paper aims to complete the relativistic study by exploring other main effects, namely, the realistic proton mass and the energy recoil of the nucleus.

The paper is structured as follows. In Section 2, we introduce some fundamental quantum models that are directly related to our subject. We describe the numerical methods in Section 3. We calculate the eigenvalues and eigenvectors for different external magnetic fields in the Sturmian basis. 
In Section 4, we introduce the effect of nucleus recoil energy and we present the results. Finally, conclusion is given in Section 5 .

\section{Model}

2.1. The Bohr Model. A succinct overview of the Bohr model which has connection to the hydrogen atom is useful and is requested $[64,65]$. This model enables us to introduce fundamental quantities such us the Bohr radius and the ionization energy. Moreover, the energies determined by Bohr theory are entirely the eigenvalues $E_{n}$ of the Hamiltonian. This semiclassical model has been established on the hypothesis that the electron describes a circular orbit of radius $r$ around the proton satisfying the following equations [55]:

$$
\begin{gathered}
E=\frac{1}{2} \mu v^{2}-\frac{e^{2}}{r}, \\
\frac{\mu v^{2}}{r}=\frac{e^{2}}{r^{2}}, \\
\mu v r=n \hbar .
\end{gathered}
$$

The term $-e^{2} / r$ of the first equation is the corresponding potential energy of the electrostatic interaction between the proton and the electron. In this expression, $e^{2}$ is written as

$$
e^{2}=\frac{q^{2}}{4 \pi \varepsilon_{0}}
$$

where $q$ is the elementary electric charge, whereas $\eta v^{2} / r$ is the kinetic energy of the relative motion of the proton and the electron. The ratio of $m_{p}$ to $m_{e}$ is close to 1800 . Thus, the reduced mass of this system is very near to $m_{e}$ :

$$
\mu=\frac{m_{p} \cdot m_{e}}{m_{p}+m_{e}} \simeq m_{e}\left(1-\frac{m_{e}}{m_{p}}\right) .
$$

In this study, we substitute the electron mass $m_{e}$ with the correction term $m_{e} / m_{p}$ into the expression of the effective mass.

In the second equation the term $e^{2} / r^{2}$ expresses the Coulomb force which is applied to the electron. The term $v^{2} / r$ is the acceleration of the electron in uniform circular motion.

To explain the existence of the stable discrete energy levels, the Bohr quantization rules were established. Besides, the energies $E_{n}$ given by this model are in agreement with quantum mechanical theory [66]:

$$
E_{n}=-\frac{m_{e} e^{4}}{2 n^{2} \hbar^{2}}
$$

where $n$ is the principal quantum number and $\hbar$ is Planck's constant.

In atomic units $\left(e=m_{e}=\hbar=1\right)$, the energy eigenvalues equation (4) becomes

$$
E_{n}=-\frac{1}{2 n^{2}}
$$

From this expression, Bohr derived the transmission wavelength between different levels of $n$, using the Rydberg constant for a nucleus of infinite mass [67]:

$$
R_{\infty}=10973731.568527 \mathrm{~m}^{-1} \text {. }
$$

The dynamic symmetry group has simplified the study of the hydrogen atom (with 6 degrees of freedom). This group is generated by the constants of motion, namely, the angular momentum $\vec{L}$, the Runge-Lenz vector $\vec{A}$ (defined by $\vec{A}=(\vec{p} \wedge$ $\vec{L}) / m-\vec{r} / r)$, and the energy $E$.

For a weak perturbation the group $\mathrm{SO} 4$ is appropriated to explain the degeneracy of the energy levels in nonrelativistic dynamics. However, for the global investigation of the discrete and continuous states, the use of the symmetry group $\mathrm{SO}(2,2)$ is required in particular for strong perturbations. In Section 3 , more details are given about the group representation of $\mathrm{SO}(2,2)$ for hydrogen atom excited by a magnetic field.

\subsection{Relativistic Corrections in an External Strong Magnetic} Field. We introduce Dirac's equation in order to take into account the relativistic effects. The energy levels are now relying on two quantum numbers: the principal quantum number $n$ and the quantum number $J$ associated with the total angular momentum of the electron $\vec{J}=\vec{L}+\vec{S}$. It can be written as follows [48]:

$$
\begin{aligned}
& E_{n J} \\
& \quad=m c^{2}\left[1+\frac{\alpha^{2}}{\left(\sqrt{(j+1 / 2)^{2}-\alpha^{2}}+n-j-1 / 2\right)^{2}}\right]^{-1 / 2},
\end{aligned}
$$

where $\alpha$ is the fine structure constant which defines the scale of the splitting:

$$
\alpha=\frac{e^{2}}{\hbar c} .
$$

The Dirac equation is generally used as a Taylor expansion in power of $\alpha^{2}$ as follows [48]:

$$
E_{n J}=-\frac{m c^{2} \alpha^{2}}{2 n^{2}}\left[1+\frac{\alpha^{2}}{n^{2}}\left(\frac{n}{J+1 / 2}-\frac{3}{4}\right)+\cdots\right] .
$$

Dependence $1 / n^{3}$ of the fine structure is immediately apparent in this expression. We studied this effect in our previous paper [63]. Dirac theory takes into account the quantum and the relativistic nature of the electron; however, it does not reflect accurately the finite mass of the nucleus. In the equations above, the mass of the nucleus is still considered infinite. To take into account the finite mass, we substitute in the Dirac equation the mass of the electron by the reduced mass $\mu=m M /(m+M)$ of the electron-proton system $(M$ is the proton mass). Furthermore we add other corrections to the energy levels obtained. The first is a relativistic correction due to the recoil of the nucleus. It is of the order of $\alpha^{2}$ with 
respect to the ground state energy of the hydrogen atom. Its expression is quite simple [48]:

$$
E_{n}(\text { recoil })=-\frac{\eta^{2} c^{2}}{M+m} \frac{\alpha^{4}}{8 n^{4}}
$$

Replacing the electron mass by the reduced mass, the expressions of the energies (4) and (10) are written in atomic unit ( $m=1, M=1836.153996)[48]$ :

$$
\begin{gathered}
E_{n}^{r}=-\left(1-\frac{m}{M}\right) \frac{1}{2 n^{2}}, \\
E_{n}(\text { recoil })=-\frac{1}{M}\left(1-\frac{3 m}{M}\right) \frac{\alpha^{2}}{8 n^{4}} .
\end{gathered}
$$

Over the last three decades, several works have been dedicated to the understanding of Rydberg atoms, highly excited hydrogen atom, in strong static fields. These studies have led to high-resolution numerical spectra as well as improving the accuracy of the calculations of the magnetic field in many problems $[48,68]$. If the contribution of the electromagnetic field compared to undisturbed energies is equal or higher, then the magnetic field is strong. In our context, chaos can be realized in the experiment with Rydberg states $(n=30,31)$.

We will study later this effect on energy diagrams. We write the Hamiltonian of hydrogen atom in a uniform magnetic field as [63]

$$
H=H_{1}+H_{p}+H_{d} \text {, }
$$

where $H_{1}, H_{p}$, and $H_{d}$ are defined as follows [66]:

$$
\begin{gathered}
H_{1}=m_{e} c^{2}+H_{0}+W_{m v}+W_{\mathrm{SO}}+W_{D}, \\
H_{p}=\frac{e^{2}}{2 m_{e}} \vec{B} \cdot(\vec{L}+2 \vec{S}), \\
H_{d}=\frac{e^{2} B^{2}}{8 m_{e}} r^{2} \sin ^{2}(\theta) .
\end{gathered}
$$

The eigenenergies of the Hamiltonian are given in (9). In this relation $m_{e} c^{2}$ is the rest-mass energy of the electron, $H_{0}=$ $p^{2} / 2 m_{e}+V(r)$ is the nonrelativistic Hamiltonian, and the following terms

$$
\begin{gathered}
W_{m v}=\frac{p^{4}}{8 m_{e}^{3} c^{2}}, \\
W_{\mathrm{SO}}=\frac{1}{2 m_{e}^{2} c^{2}} \frac{1}{r} \frac{d V(r)}{d r} \vec{L} \cdot \vec{S}, \\
W_{D}=\frac{\hbar^{2}}{8 m_{e}^{2} c^{2}} \Delta V(r)
\end{gathered}
$$

describe the fine structure. $\vec{L}$ and $\vec{S}$ represent, respectively, the orbital and spin moments of the atom, $\vec{r}$ is the radius-vector of the atomic electron, and $\theta$ designs the angle between $\vec{r}$ and $\vec{B}$.

The paramagnetic term $H_{p}$ is the origin of the linear Zeeman effect offering the splitting of the atomic levels, while the diamagnetic energy term $H_{d}$ is responsible for the complex nature of the problem.

Since $L_{z}$ is a constant of the motion, the linear Zeeman energy $H_{p}$ plays no significant role in the dynamics [68]. For high $n$ levels, the diamagnetic term rapidly dominates the paramagnetic one, and it may even become comparable to or greater than the Coulomb term itself, leading to a complete modification of the structure of atomic spectra. In fact the linear Zeeman (paramagnetic) term is independent of $n$, while the diamagnetic term is proportional to $n^{4}$. The hydrogen atom Hamiltonian becomes nonseparable when the diamagnetic term is included $[68,69]$.

In this paper we are studying the effect of the diamagnetic term $H_{d}$ (in atomic units $H_{d}=\left(B^{2} / 8\right) r^{2} \sin ^{2}(\theta)$ ) on the Rydberg atom. $H_{d}$ is responsible for the chaos manifestation.

As an important criterion, chaos appears when the value of the diamagnetic term is comparable to the unperturbed energies $[59,68]$.

\section{Computation Method}

All computed spectra presented in this paper are obtained by digitalization of the Hamiltonian matrix in a suitable basis. It is worth noting that the spherical Sturmian basis has been applied for the study of hydrogen atom in a magnetic field. Our simulations are performed by developing a code based on MAPLE in which we can add new correction effects. It is advantageous to choose the Sturmian basis well adopted to dynamic symmetries and it does not saturate the computer memory.

3.1. Spherical Sturmian Basis. Computing the spectra of diamagnetic hydrogen in a spherical Sturmian basis was firstly used by Edmonds [70]. Clark and Taylor [68] recognized the importance of being able to shift the length scale of the basis relying on the region of energy and field. Delande applied a complex rotation to the Sturmian basis to compute the spectrum of the diamagnetic hydrogen for positive energy, being aware that this technique has already been extended to other atoms [38].

Efficient calculations are provided by this basis choice which needs several considerations of symmetry [71]. All hydrogenoid states that share the same value $M$ are mixed; therefore, it is a need to consider a dynamic group of diverse states. It is acknowledgeable that the group $\mathrm{SO}(2,2)$ applies the previous criterion. In fact, this group is founded on the eigenstates that are themselves the basis of the Sturmian functions. On one hand, this basis enables the global representation of states' space (discrete, continuum). On the other hand, it results in the loss of orthogonality of the basis vectors.

The Sturmian basis is the proper basis describing the system of two oscillators equivalent to the Rydberg atom. We 
are able to express the Schrödinger equation in function of these generators $\left(\vec{S}^{(\alpha)}, \vec{T}^{(\alpha)}\right)$ of dynamic group $\mathrm{SO}(2,2)$. They are defined, respectively, on an adjustable parameter $\alpha$ [38]:

$$
\begin{aligned}
& S_{1}^{(\alpha)}=-\frac{\alpha}{4}\left(p_{\eta}^{2}+\frac{L_{z}^{2}}{\eta^{2}}\right)+\frac{1}{4 \alpha} \eta^{2}, \\
& S_{2}^{(\alpha)}=-\frac{1}{2} \eta p_{\eta}, \\
& S_{3}^{(\alpha)}=\frac{\alpha}{4}\left(p_{\eta}^{2}+\frac{L_{z}^{2}}{\eta^{2}}\right)+\frac{1}{4 \alpha} \eta^{2}, \\
& T_{1}^{(\alpha)}=-\frac{\alpha}{4}\left(p_{\nu}^{2}+\frac{L_{z}^{2}}{v^{2}}\right)+\frac{1}{4 \alpha} v^{2}, \\
& T_{3}^{(\alpha)}=\frac{\alpha}{4}\left(p_{\nu}^{2}+\frac{L_{z}^{2}}{v^{2}}\right)+\frac{1}{4 \alpha} v^{2},
\end{aligned}
$$

where semiparabolic coordinates $(\eta, \nu)$ are defined as

$$
\begin{aligned}
& \eta=\sqrt{r+z} \\
& \nu=\sqrt{r-z}
\end{aligned}
$$

while the momentums $\left(p_{\eta}, p_{\nu}\right)$ are defined as

$$
\begin{aligned}
& p_{\eta}=\left(\eta^{2}+v^{2}\right) \frac{d \eta}{d t} \\
& p_{\nu}=\left(\eta^{2}+\nu^{2}\right) \frac{d \nu}{d t}
\end{aligned}
$$

and $L_{z}$ is the $z$-component of the angular momentum.

These 6 variables are not independent:

$$
\begin{aligned}
& \vec{S}^{2}=\left(S_{3}^{(\alpha)}\right)^{2}-\left(S_{1}^{(\alpha)}\right)^{2}-\left(S_{2}^{(\alpha)}\right)^{2}=\frac{L_{z}^{2}}{4}, \\
& \vec{T}^{2}=\left(T_{3}^{(\alpha)}\right)^{2}-\left(T_{1}^{(\alpha)}\right)^{2}-\left(T_{2}^{(\alpha)}\right)^{2}=\frac{L_{z}^{2}}{4} .
\end{aligned}
$$

Thus the dynamic variables $\left(\vec{S}^{(\alpha)}, \vec{T}^{(\alpha)}\right)$ represent the classical form of the quantum generator of the dynamic group $\mathrm{SO}(2,2)$.

The Hamiltonian "oscillator" obtained coincides with its expression in the context of the classic mechanic:

$$
\begin{aligned}
& \left(S_{3}^{(\alpha)}+T_{3}^{(\alpha)}+\frac{\gamma^{2}}{2(-2 E)^{2}}\left(S_{1}^{(\alpha)}+S_{3}^{(\alpha)}\right)\left(T_{1}^{(\alpha)}+T_{3}^{(\alpha)}\right)\right. \\
& \left.\times\left(S_{1}^{(\alpha)}+S_{3}^{(\alpha)}+T_{1}^{(\alpha)}+T_{3}^{(\alpha)}\right)-\frac{1}{\sqrt{-2 E}}\right)|\Psi\rangle=0,
\end{aligned}
$$

with $\alpha=1 / \sqrt{-2 E}$.
We can write a more general equation by taking any value of the adjustable parameter $\alpha$ [57]. We find

$$
\left[A^{(\alpha)}-\alpha+\left(-2 E \alpha^{2}\right) B^{(\alpha)}+\frac{\gamma^{2} \alpha^{4}}{2} C^{(\alpha)}\right]|\Psi\rangle=0
$$

with

$$
\begin{gathered}
A^{(\alpha)}=\frac{S_{3}^{(\alpha)}-S_{1}^{(\alpha)}}{2}+\frac{T_{3}^{(\alpha)}-T_{1}^{(\alpha)}}{2}, \\
B^{(\alpha)}=\frac{S_{3}^{(\alpha)}+S_{1}^{(\alpha)}}{2}+\frac{T_{3}^{(\alpha)}+T_{1}^{(\alpha)}}{2}, \\
C^{(\alpha)}=\left(S_{1}^{(\alpha)}+S_{3}^{(\alpha)}\right)\left(T_{1}^{(\alpha)}+T_{3}^{(\alpha)}\right)\left(S_{1}^{(\alpha)}+S_{3}^{(\alpha)}+T_{1}^{(\alpha)}+T_{3}^{(\alpha)}\right) .
\end{gathered}
$$

In the Sturmian basis, the eigenvalues of the hydrogen Hamiltonian are obtained by solving the generalized eigenvalue problem (20).

The spherical Sturmian basis functions are [72]

$$
\Psi_{n l m}(r)=S_{n l}^{(\alpha)}(r) Y_{l m}(\theta, \varphi)
$$

where $S_{n l}^{(\alpha)}(r)$ is the radial Sturmian function:

$$
S_{n l}^{(\alpha)}(r)=\left[\frac{(n-l-1) !}{2(n+l) !}\right]^{(1 / 2)}\left(\frac{r}{2}\right)^{l+1} e^{-r / 2 \alpha} L_{n-l-1}^{2 l+1}\left(\frac{r}{\alpha}\right),
$$

and $L_{i}^{j}$ is the associated Laguerre polynomial.

The full eigenfunctions used can be written as

$$
|n l m\rangle=S_{n l}^{(\alpha)} Y_{l m}
$$

Then, the matrix element of the diamagnetic term $\left(B^{2} / 8\right) r^{2} \sin ^{2}(\theta)$ is given by

$$
\begin{aligned}
\left\langle n^{\prime} l^{\prime} m\left|\frac{B^{2}}{8} r^{2} \sin ^{2}(\theta)\right| n l m\right\rangle= & \frac{1}{8} B^{2}\left\langle n^{\prime} l^{\prime}\left|r^{2}\right| n l\right\rangle \\
& \times\left\langle l^{\prime} m\left|\sin ^{2}(\theta)\right| l m\right\rangle .
\end{aligned}
$$

Evaluation of the angular matrix elements is then easy. The corresponding matrix elements of the square position operator $r^{2}$ are written as [68]

$$
\left\langle n^{\prime} l^{\prime}\left|r^{2}\right| n l\right\rangle=\int_{0}^{\infty} S_{n l}^{\zeta}(r) r^{2} S_{n^{\prime} l^{\prime}}^{\zeta}(r) d r
$$


are nonzero only for $\left|n-n^{\prime}\right|=0,1,2,3,\left|l-l^{\prime}\right|=0,2$, and at these points take the following forms:

$$
\begin{aligned}
& \left\langle n^{\prime} l+2\left|r^{2}\right| n l\right\rangle
\end{aligned}
$$

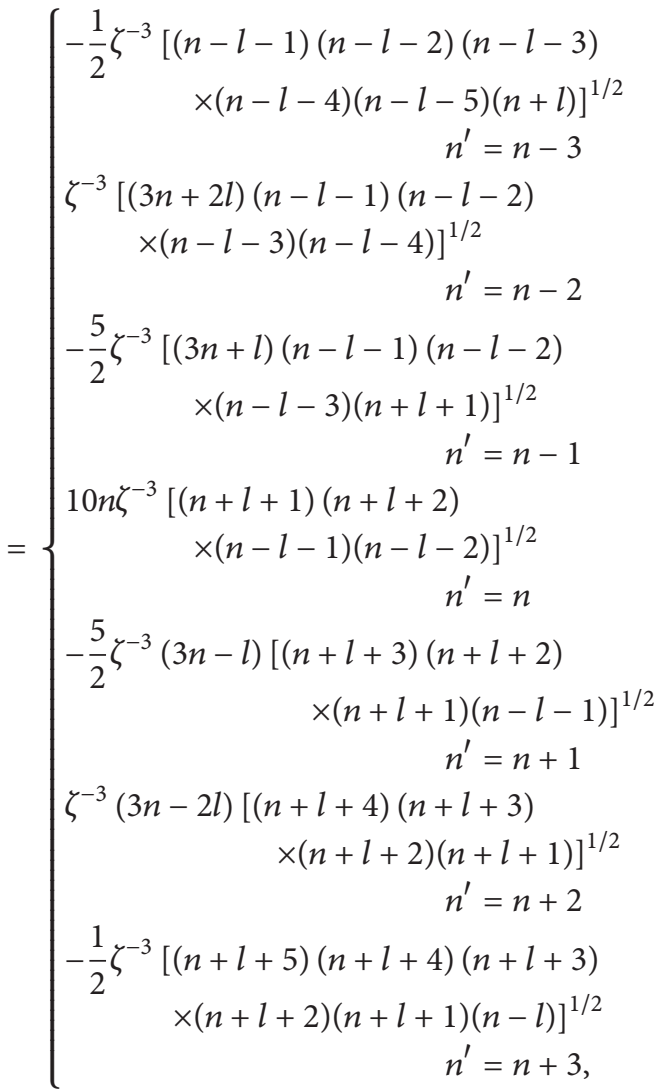

$$
\begin{aligned}
& \left\langle n^{\prime} l\left|r^{2}\right| n l\right\rangle \\
& \left\{\begin{array}{c}
2 n \zeta^{-3}\left[5 n^{2}-3 l(l+1)+1\right] \quad n^{\prime}=n \\
-\frac{3}{2} \zeta^{-3}[5 n(n+1)-l(l+1)+2] \\
\times[(n+l+1)(n-l)]^{1 / 2} \\
n^{\prime}=n+1 \\
3 \zeta^{-3}(n+1)[(n+l+1)(n-l) \\
\times(n+l+2)(n-l+1)]^{1 / 2} \\
n^{\prime}=n+2 \\
-\frac{1}{2} \zeta^{-3}[(n+l+1)(n+2-l)(n-l) \\
\times(n+l+3)(n+l+2)(n+1-l)]^{1 / 2} \\
n^{\prime}=n+3 .
\end{array}\right.
\end{aligned}
$$

The investigation of Clark and Taylor [68] has revealed that it is usually advisable to select higher values of $\zeta$; In our simulation we used $\zeta=2 / n$ in order to be close to the ionization limit.

Our numerical simulations consist in diagonalizing the matrix representing the operator in a Sturmian basis. The matrix elements of the generators are independent of $\alpha$.
3.2. Numerical Method. Equation (20) is equivalent to the general problem for eigenvalues of the form [59]

$$
(M-\lambda N)|\Psi\rangle=0,
$$

where $M$ and $N$ are fixed matrices and $\lambda$ and $|\Psi\rangle$ are the eigenvalues and the eigenvectors searched.

3.2.1. Simulation at Fixed Magnetic Field. In the case where we fix the magnetic field $\gamma=B / B_{c}$ ( $\gamma$ denotes the magnetic field in units of $B_{c} \simeq 2.35 \times 10^{5} \mathrm{~T}$ ) after opting for $\alpha=\alpha_{0}=$ $C^{t e}$, we obtain an equation in the generalized eigenvalues with [59]

$$
\begin{gathered}
M=A^{(\alpha)}-\alpha_{0}+\frac{\gamma^{2} \alpha_{0}^{4}}{2} C^{(\alpha)}, \\
N=B^{(\alpha)}, \\
\lambda=-2 E \alpha_{0}^{2}
\end{gathered}
$$

that determines the energy levels.

3.2.2. Simulation at Fixed Energy. In this simulation we choose $\alpha=\alpha_{0}=C^{\text {te }}$ and we fix the energy. So that we get an equation in the generalized eigenvalues with [59]

$$
\begin{gathered}
M=A^{(\alpha)}-\alpha_{0}+\left(-2 E \alpha_{0}^{2}\right) B^{(\alpha)}, \\
N=C^{(\alpha)} \\
\lambda=\frac{\gamma^{2} \alpha_{0}^{4}}{2}
\end{gathered}
$$

that establishes the spectrum of magnetic field values related to the fixed energy.

Both types of the simulations give the same outcomes.

\subsubsection{Diagonalization Algorithm and Convergence Criterion.} The matrix components of the generators $\left(\vec{S}^{(\alpha)}, \vec{T}^{(\alpha)}\right)$ in a Sturmian basis are restricted to some selections' rules. It is well known that the matrix eigenvalue problems, generally to be solved, are real symmetric matrices in strips [38].

Currently, stable numerical algorithms have been developed in order to calculate the eigenvalues and eigenvectors [48].

We provide an adoptable parameter $\alpha_{0}$ to verify the convergence for each type of the simulations. In fact, $\alpha_{0}$ is connected to the natural frequency of the basic oscillator selected. The accuracy of the examined values is not reliant on this parameter. Exact searched values are not dependent on this parameter.

\section{Numerical Results and Discussion}

4.1. Energy Diagrams. We use the simulation at fixed magnetic field and we plot for a large number of points diagrams $E_{n}^{r}=f\left(\gamma^{2}\right)$ in Figures 1, 2, and 3 .

Figure 1 shows the diagram $E$ (Hartree) $=f\left(\gamma^{2}\right)$ for Rydberg states $n=30$ in the system with rather low field 


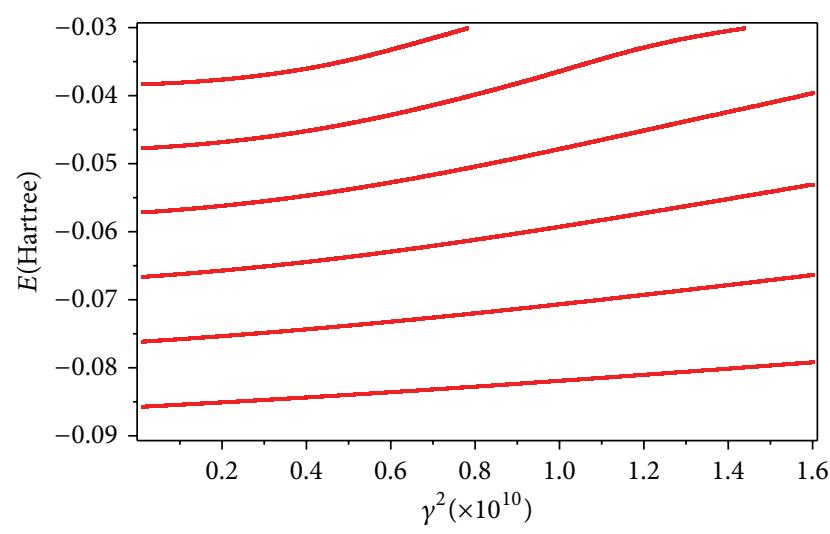

FIGURE 1: Energy levels as a function of the normalized square magnetic field $\gamma^{2}\left(\gamma=B / B_{c}, B_{c}=2.35 \times 10^{5} T\right)$ calculated by the simulation technique described in Section 3.2.1 for weak magnetic field $B(B<3 T)$ and for the principle quantum number $n=30$. The rovibration structure is completely removed (inter-l-mixing). We observe that, for a weak magnetic field, the classical dynamics is regular.

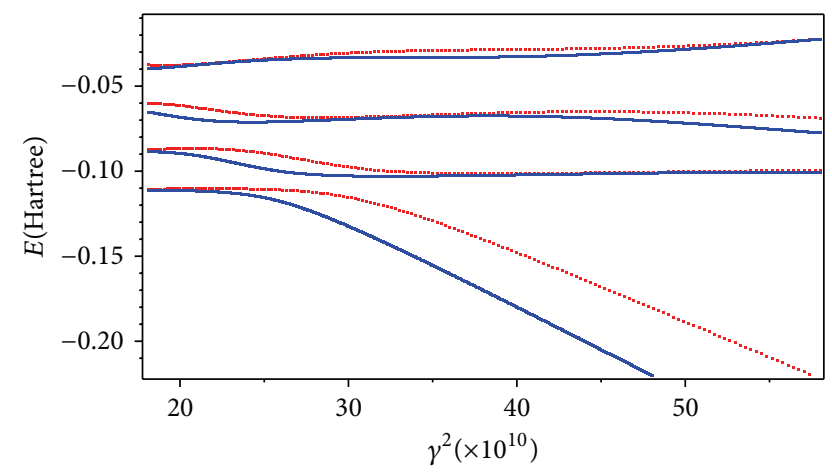

FIgURE 2: Diagram of energy levels of the hydrogen atom in strong magnetic field as function of $\gamma^{2}\left(\gamma=B / B_{c}, B_{c}=2.35 \times\right.$ $10^{5} \mathrm{~T}$ ) computed with a simulation described in Section 3.2.1. The calculation is done for a strong magnetic field $B(9 T<B<18 T)$ for both levels $n=30$ (dotted red line) and $n=31$ (solid blue line). Mixing between the energy levels of $n=30$ and $n=31$ is observed.

$(B<3 T)$ : diamagnetism breaks the zero-field $l$-degeneracy of the hydrogen atom.

We can explain this by the fact that, in very weak field (l-system intermixing), the degeneracy of the levels (the rovibrational structure) proportional to $\gamma^{2}$ is completely removed by the diamagnetic term. It is visible that regular system disturbed by a weak perturbation remains essentially regular for the reason that the diamagnetic interaction is a perturbation of the Coulomb dynamics. That is a regular regime: there is no sensitivity on initial conditions.

At high magnetic field, as shown by Figure 2, the different energy levels $(n=30, n=31)$ essentially cross.

Indeed for a highly excited state with principal quantum number $n$, the diamagnetic interaction scales as $\gamma^{2} n^{4}$ and

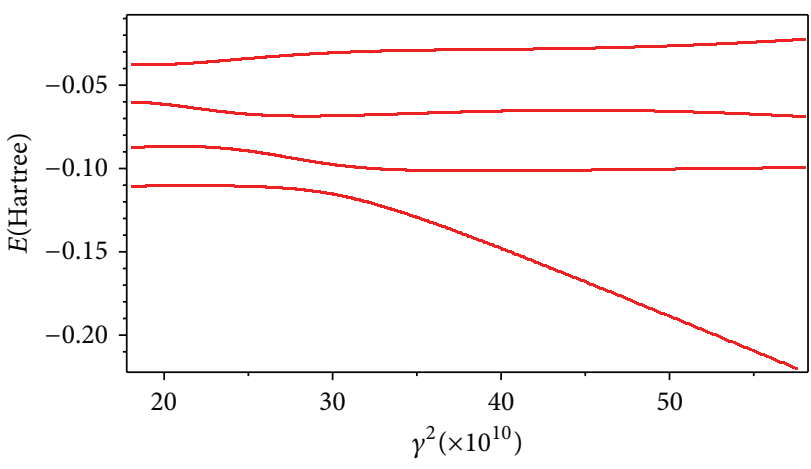

FIGURE 3: Diagram of the energy levels of the hydrogen atom in the strong magnetic field for $n=30$ computed by the simulation described in Section 3.2.1. We note that for a high magnetic field the energy levels anticross. This is an indication of the chaos emergence.

the zero-field as $1 / n^{2}$. The strong field regime is obtained when [38]

$$
\gamma n^{3} \simeq 1, \quad \text { that is } B n^{3} \simeq B_{c} .
$$

This is achieved with $n \simeq 30$ and $B \simeq 9 T$. The rotational and vibrational energy levels really cross. By increasing the magnetic field, in Figure 3, we observe that more states anticross. The classical smooth transition from regularity to chaos agrees well with this result.

4.2. The Effect of the Nucleus Recoil Energy. In this section, we use the second simulation, simulation at fixed energy, where we have plotted $\gamma^{2}$ as a function of the angular momentum $l$. We introduced the recoil energy effect given, in atomic unit, by

$$
E_{n}(\text { recoil })=-\frac{1}{M}\left(1-\frac{3 m}{M}\right) \frac{\alpha^{2}}{8 n^{4}},
$$

which takes into account the relativistic effects. we recall that the levels shift due to this effect can be written as [68]

$$
\Delta E_{l}=\frac{\alpha^{2}}{2 n^{3} l(l+1)} .
$$

This recoil energy has been introduced with which we have realized the simulation with nucleus of finite mass. The result of the simulation is shown in Figure 4, where the distinction between the nucleus having a finite mass (diamond line) and the one having an infinite mass (circle line) is very clear. This difference can be justified by the fact that the term (33) is the amount of three contributions. The first contribution is the kinetic energy in which the electron mass and not the reduced mass of the system manifests

$$
W_{m v}=\frac{\vec{P}^{4}}{8 m_{e}^{3} c^{2}} .
$$

The second contribution is the Darwin term which takes into account the electron delocalization but not the nucleus:

$$
\frac{\hbar^{2}}{8 m_{e}^{2} c^{2}} 4 \pi e^{2} \delta(\vec{r}) \text {. }
$$




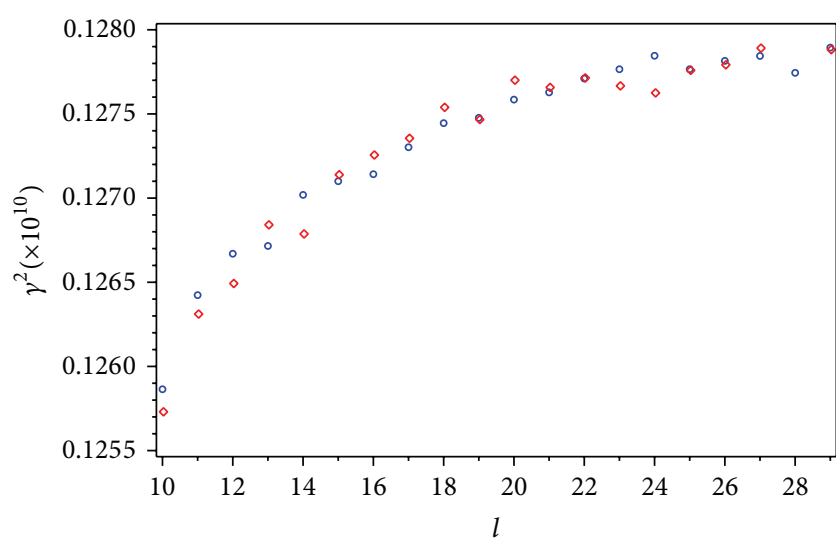

FIGURE 4: The normalized square magnetic field $\gamma^{2}\left(\gamma=B / B_{c}\right.$, $\left.B_{c}=2.35 \times 10^{5} \mathrm{~T}\right)$ for different angular momentum 1 with a principal quantum $n=30$ calculated by the simulation described in Section 3.2.2. Two cases are computed: first case: realistic nucleus mass (finite mass) (red diamond line); second case: nucleus infinite mass (blue circle line).

Finally, the third contribution is the spin-orbit which shows the interaction between the electron magnetic moment and the magnetic field of the nucleus seen in the proper frame of the electron. Therefore we should take into consideration the nucleus speed since the nucleus of finite mass is being in motion.

\section{Conclusion}

We have studied the diamagnetic behavior of a hydrogen atom highly excited in a strong magnetic field considering the realistic finite mass of the nucleus. The results reveal that when the magnetic field is weak, the diamagnetic term removes the degeneracy of the energy levels. The system is totally regular: it is possible to affect quantum numbers to the various energy levels. However, with increasing magnetic field any symmetry will be destroyed and the energy levels will be crossed; there is an unruffled transition from regularity to chaotical dynamics. At higher magnetic field, the dynamic is totally chaotic and quantum spectrum includes only large anticrossing.

\section{Conflict of Interests}

The authors declare that there is no conflict of interests regarding the publication of this paper.

\section{References}

[1] B. Li and H. P. Liu, "An effective quantum defect theory for the diamagnetic spectrum of a barium Rydberg atom," Chinese Physics B, vol. 22, no. 1, Article ID 013203, 2013.

[2] A. E. Shabad and V. V. Usov, "Modified coulomb law in a strongly magnetized vacuum," Physical Review Letters, vol. 98, no. 18, Article ID 180403, 2007.

[3] L. Stevanovic and D. Milojevic, "Compressed hydrogen atom under debye screening in strong magnetic field," Publications of the Astronomical Observatory of Belgrade, vol. 91, pp. 327-330, 2012.

[4] H. Jabri, H. Eleuch, and T. Djerad, "Lifetimes of atomic Rydberg states by autocorrelation function," Laser Physics Letters, vol. 2, no. 5, pp. 253-257, 2005.

[5] H. Eleuch and N. Rachid, "Autocorrelation function of microcavity-emitting field in the non-linear regime," The European Physical Journal D, vol. 57, no. 2, pp. 259-264, 2010.

[6] H. Eleuch, N. Ben Nessib, and R. Bennaceur, "Quantum model of emission in a weakly non ideal plasma," The European Physical Journal D, vol. 29, no. 3, pp. 391-395, 2004.

[7] P. K. Jha, H. Eleuch, and Y. V. Rostovtsev, "Coherent control of atomic excitation using off-resonant strong few-cycle pulses," Physical Review A, vol. 82, no. 4, Article ID 045805, 2010.

[8] E. A. Sete and H. Eleuch, "Strong squeezing and robust entanglement in cavity electromechanics," Physical Review A, vol. 89, no. 1, Article ID 013841, 2014.

[9] M. Khatua, U. Sarkar, and P. K. Chattaraj, "Reactivity dynamics of confined atoms in the presence of an external magnetic field," The European Physical Journal D, vol. 68, no. 2, article 22, 2014.

[10] U. Das and B. Mukhopadhyay, "New mass limit for white dwarfs: super-Chandrasekhar type Ia supernova as a new standard candle," Physical Review Letters, vol. 110, no. 7, Article ID 071102, 2013.

[11] A. D. Sargsyan, R. K. Mirzoyan, A. S. Sarkisyan, A. H. Amiryan, and D. H. Sarkisyan, "Splitting of N-type optical resonance formed in $\Lambda$-system of ${ }^{85} \mathrm{Rb}$ atoms in a strong transverse magnetic field," Journal of Contemporary Physics, vol. 49, no. 1, pp. 20-27, 2014.

[12] V. S. Popov and B. M. Karnakov, "Hydrogen atom in a strong magnetic field," Physics-Uspekhi, vol. 57, no. 3, pp. 257-279, 2014.

[13] E. Giacobino, J.-P. Karr, G. Messin, H. Eleuch, and A. Baas, "Quantum optical effects in semiconductor microcavities," Comptes Rendus Physique, vol. 3, no. 1, pp. 41-52, 2002.

[14] R. Juárez-Amaro, A. Zúñiga-Segundo, F. Soto-Eguibar, and H. M. Moya-Cessa, "Equivalence between mirror-field-atom and ion-laser interactions," Applied Mathematics \& Information Sciences, vol. 7, no. 4, pp. 1311-1315, 2013.

[15] H. Eleuch, S. Guérin, and H. R. Jauslin, "Effects of an environment on a cavity-quantum-electrodynamics system controlled by bichromatic adiabatic passage," Physical Review A, vol. 85, no. 1, Article ID 013830, 2012.

[16] E. A. Sete, A. A. Svidzinsky, Y. V. Rostovtsev et al., "Using quantum coherence to generate gain in the xuv and $\mathrm{X}$-ray: gain-swept superradiance and lasing without inversion," IEEE Journal on Selected Topics in Quantum Electronics, vol. 18, no. 1, pp. 541-553, 2012.

[17] E. A. Sete, A. A. Svidzinsky, H. Eleuch, Z. Yang, R. D. Nevels, and M. O. Scully, "Correlated spontaneous emission on the Danube," Journal of Modern Optics, vol. 57, no. 14-15, pp. 13111330, 2010 .

[18] Z. B. Walters, "Quantum coherent dynamics at ambient temperature in photosynthetic molecules," Quantum Physics Letters, vol. 1, no. 1, pp. 21-33, 2012.

[19] H. Rabitz, "Control in the sciences over vast length and time scales," Quantum Physics Letters, vol. 1, no. 1, pp. 1-19, 2012.

[20] D. A. Barskiy, K. V. Kovtunov, I. V. Koptyug et al., “The feasibility of formation and kinetics of NMR signal amplification by reversible exchange (SABRE) at high magnetic field (9.4 T)," Journal of the American Chemical Society, vol. 136, no. 9, pp. 3322-3325, 2014. 
[21] B. Mandal, S. Adhikari, K. Choudhary et al., "Femtosecond response of quantum discrete breathers in SRR based metamaterials and the role of dielectric permittivity," Quantum Physics Letters, vol. 1, no. 2, pp. 59-68, 2012.

[22] J. D. Pritchard, J. A. Isaacs, M. A. Beck, R. McDermott, and M. Saffman, "Hybrid atom-photon quantum gate in a superconducting microwave resonator," Physical Review A, vol. 89, no. 1, Article ID 010301, 2014.

[23] P. Rej and A. Ghoshal, "Rydberg transitions for positronhydrogen collisions: asymptotic cross section and scaling law," Journal of Physics B: Atomic, Molecular and Optical Physics, vol. 47, no. 1, Article ID 015204, 2014.

[24] H. Eleuch, D. Elser, and R. Bennaceur, "Soliton propagation in an absorbing three-level atomic system," Laser Physics Letters, vol. 1, no. 8, pp. 391-396, 2004.

[25] H. Eleuch and R. Bennaceur, "An optical soliton pair among absorbing three-level atoms," Journal of Optics A: Pure and Applied Optics, vol. 5, no. 5, pp. 528-533, 2003.

[26] E. Osman, M. Khalfallah, and H. Sapoor, "Improved ( $\left.G^{\prime} / G\right)$ expansion method for elliptic-like equation and general traveling wave solutions of some class of NLPDEs," Information Science Letters, vol. 2, no. 2, pp. 123-127, 2013.

[27] M. Akbari, "Exact solutions of the coupled Higgs equation and the Maccari system using the modified simplest equation method," Information Science Letters, vol. 2, no. 3, pp. 155-158, 2013.

[28] N. Taghizadeh, M. Mirzazadeh, M. Akbari, and M. Rahimian, "Exact soliton solutions for generalized equal width equation," Mathematical Science Letters, vol. 2, no. 2, pp. 99-106, 2013.

[29] E. Osman, M. Khalfallah, and H. Sapoor, "Finding general solutions of nonlinear evolution equations by improved ( $\left.G^{\prime} / G\right)$ expansion method," Mathematical Science Letters, vol. 3, no. 1, pp. 1-8, 2014.

[30] E. M. E. Zayed, "Exact traveling wave solutions for a variablecoefficient generalized dispersive water-wave system using the generalized (G'/G)-expansion method," Mathematical Science Letters, vol. 3, no. 1, pp. 9-15, 2014.

[31] E. A. Sete and H. Eleuch, "Interaction of a quantum well with squeezed light: quantum-statistical properties," Physical Review A, vol. 82, no. 4, Article ID 043810, 2010.

[32] W. Yang, L. Huang, and M. Tian, "Two novel and secure quantum ANDOS schemes," Applied Mathematics and Information Sciences, vol. 7, no. 2, pp. 659-665, 2013.

[33] H. Eleuch, "Photon statistics of light in semiconductor microcavities," Journal of Physics B: Atomic, Molecular and Optical Physics, vol. 41, no. 5, Article ID 055502, 2008.

[34] G. Messin, J. P. Karr, H. Eleuch, J. M. Courty, and E. Giacobino, "Squeezed states and the quantum noise of light in semiconductor microcavities," Journal of Physics Condensed Matter, vol. 11, no. 31, pp. 6069-6078, 1999.

[35] A. Baas, J. P. Karr, H. Eleuch, and E. Giacobino, "Optical bistability in semiconductor microcavities," Physical Review A: Atomic, Molecular, and Optical Physics, vol. 69, no. 2, 2004.

[36] J. Bonca and S. Kruchinin, Physical Properties of Nanosystems, Springer, 2011.

[37] L. Tang and J.-A. Lu, "Projective lag synchronization of chaotic systems with parameter mismatch," Applied Mathematics \& Information Sciences, vol. 7, no. 1, pp. 121-125, 2013.

[38] D. Delande, "Chaos and atoms in strong magnetic fields," Physica Scripta, vol. 1991, no. T34, p. 52, 1991.
[39] H. Ruder, G. Wunner, H. Herold, and F. Geyer, Atoms in Strong Magnetic Fields, Springer, Berlin, Germany, 1994.

[40] H. Eleuch and A. Prasad, "Chaos and regularity in semiconductor microcavities," Physics Letters A, vol. 376, no. 26-27, pp. 19701977, 2012.

[41] A. Y. Potekhin and A. V. Turbiner, "Hydrogen atom in a magnetic field: the quadrupole moment," Physical Review A: Atomic, Molecular, and Optical Physics, vol. 63, no. 6, Article ID 065402, 2001.

[42] S. N. Rasband, Chaotic Dynamics of Non linear Systems, John Wiley \& Sons, 1990.

[43] A. J. Lichtenberg and M. A. Lieberman, Regular and Stochastic Motion, Springer, New York, NY, USA, 1983.

[44] A. V. Andreev, O. Agam, B. D. Simons, and B. L. Altshuler, "Quantum chaos, irreversible classical dynamics, and random matrix theory," Physical Review Letters, vol. 76, no. 21, pp. 39473950, 1996.

[45] O. Bohigas, "Les Houches summer school, session LII," in Chaos and Quantum Physics, M.-J. Giannoni, A. Voros, and J. ZinnJustin, Eds., North-Holland, Amsterdam, The Netherlands, 1991.

[46] T. Pohl, H. R. Sadeghpour, and P. Schmelcher, "Cold and ultracold Rydberg atoms in strong magnetic fields," Physics Reports, vol. 484, no. 6, pp. 181-229, 2009.

[47] T. F. Gallagher, Rydberg Atoms, Cambridge University Press, Cambridge, UK, 1994.

[48] M. I. Eides, H. Grotch, and V. A. Shelyuto, Theory of Light Hydrogenic Bound States, vol. 222 of Springer Tracts in Modern Physics, 2007.

[49] Y.-J. Liu, S. Tong, and C. L. P. Chen, "Adaptive fuzzy control via observer design for uncertain nonlinear systems with unmodeled dynamics," IEEE Transactions on Fuzzy Systems, vol. 21, no. 2, pp. 275-288, 2013.

[50] C. Yang, S. S. Ge, and T. H. Lee, "Output feedback adaptive control of a class of nonlinear discrete-time systems with unknown control directions," Automatica, vol. 45, no. 1, pp. 270276, 2009.

[51] Y.-J. Liu, C. L. P. Chen, G.-X. Wen, and S. Tong, "Adaptive neural output feedback tracking control for a class of uncertain discrete-time nonlinear systems," IEEE Transactions on Neural Networks, vol. 22, no. 7, pp. 1162-1167, 2011.

[52] C. Yang, S. S. Ge, C. Xiang, T. Chai, and T. H. Lee, "Output feedback NN control for two classes of discrete-time systems with unknown control directions in a unified approach," IEEE Transactions on Neural Networks, vol. 19, no. 11, pp. 1873-1886, 2008.

[53] Y.-J. Liu, S.-C. Tong, D. Wang, T.-S. Li, and C. L. P. Chen, "Adaptive neural output feedback controller design with reducedorder observer for a class of uncertain nonlinear SISO Systems," IEEE Transactions on Neural Networks, vol. 22, no. 8, pp. 13281334, 2011.

[54] T. M. Shahwan, "A comparison of Bayesian methods and artificial neural networks for forecasting chaotic financial time series," Journal of Statistics Applications \& Probability, vol. 1, no. 2, p. 89, 2012.

[55] J. W. Series, The Spectrum of Atomic Hydrogen, Oxford University Press, London, UK, 1957.

[56] T. van der Veldt, W. Vassen, and W. Hogervorst, "Helium Rydberg states in parallel electric and magnetic fields," Journal of Physics B: Atomic, Molecular and Optical Physics, vol. 26, no. 13, pp. 1945-1955, 1993. 
[57] A. Arimondo, W. D. Phillips, and F. Strumia, Laser Manipulation of Atoms and Ions, North-Holland, Amsterdam, The Netherlands, 1992.

[58] D. Weiss, K. Richter, A. Menschig et al., "Quantized periodic orbits in large antidot arrays," Physical Review Letters, vol. 70, no. 26, pp. 4118-4121, 1993.

[59] D. Delande, Atomes de rydberg en champs statiques intenses [Thèse d'Etat], Université Paris 6, Paris, France, 1988.

[60] M. Sadhukhan and B. M. Deb, "A dynamical signature of quantum chaos in hydrogen atom under strong, oscillating magnetic fields," EPL, vol. 94, no. 5, Article ID 50008, 2011.

[61] A. Bruno-Alfonso, C. Bleasdale, G. V. B. de Souza, and R. A. Lewis, "Closed-orbit dependence on the field direction in the anisotropic diamagnetic Kepler problem," Physical Review AAtomic, Molecular, and Optical Physics, vol. 89, no. 4, Article ID 043425, 2014.

[62] J.-K. Xu, H.-Q. Chen, and H.-P. Liu, "Exact quantum defect theory approach for lithium in magnetic fields," Chinese Physics B, vol. 22, no. 1, Article ID 013204, 2013.

[63] M. A. Amdouni and H. Eleuch, "Influence of relativistic terms in the spectra of hydrogen atom highly excited in an external strong magnetic field," Applied Mathematics \& Information Sciences, vol. 5, no. 2, pp. 307-320, 2011.

[64] J. C. Slater, Quantum Theory of Atomic Structure, vol. 1-2, McGraw-Hill, New York, NY, USA, 1996.

[65] E. U. Condon and G. H. Shortley, The Theory of Atomic Spectra, Cambridge University Press, Cambridge, UK, 1953.

[66] C. C. Tannoudji, B. Diu, and F. Laloe, Quantum Mechanics, Wiley, New York, NY, USA, 1977, Mechanics, Wiley,.

[67] P. J. Mohr, B. N. Taylor, and D. B. Newell, "CODATA recommended values of the fundamental physical constants: 2006," Reviews of Modern Physics, vol. 80, no. 2, pp. 633-730, 2008.

[68] C. W. Clark and K. T. Taylor, "The quadratic Zeeman effect in hydrogen Rydberg series: application of Sturmian functions," Journal of Physics B: Atomic and Molecular Physics, vol. 15, no. 8, pp. 1175-1193, 1982.

[69] M. A. Iken, F. Borondo, R. M. Benito, and T. Uzer, "Periodicorbit spectroscopy of the hydrogen atom in parallel electric and magnetic fields," Physical Review A, vol. 49, no. 4, pp. 2734-2747, 1994.

[70] A. R. Edmonds, "Studies of the quadratic Zeeman effect. I. Application of the sturmian functions," Journal of Physics B: Atomic and Molecular Physics, vol. 6, no. 8, pp. 1603-1615, 1973.

[71] K. Sacha, J. Zakrzewski, and D. Delande, "Breaking time reversal symmetry in chaotic driven rydberg atoms," Annals of Physics, vol. 283, no. 1, pp. 141-172, 2000.

[72] M. Courtney, Rydberg atoms in strong fields: a testing ground for quantum chaos [Ph.D. thesis], Department of Physics, Massachusetts Institute of Technology, 1995. 


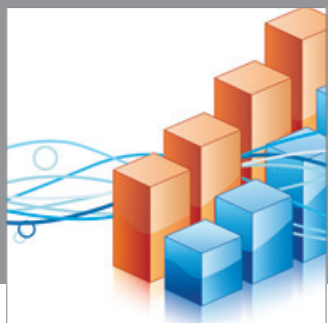

Advances in

Operations Research

mansans

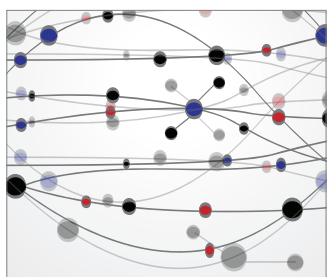

The Scientific World Journal
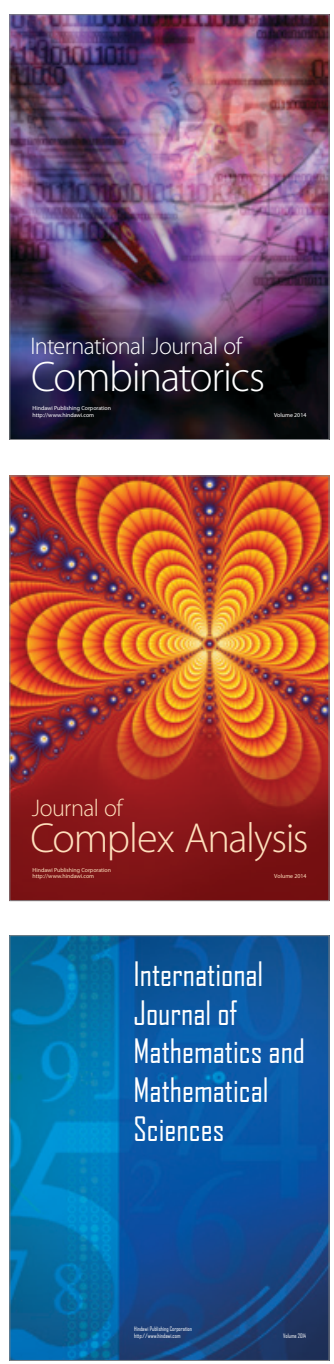
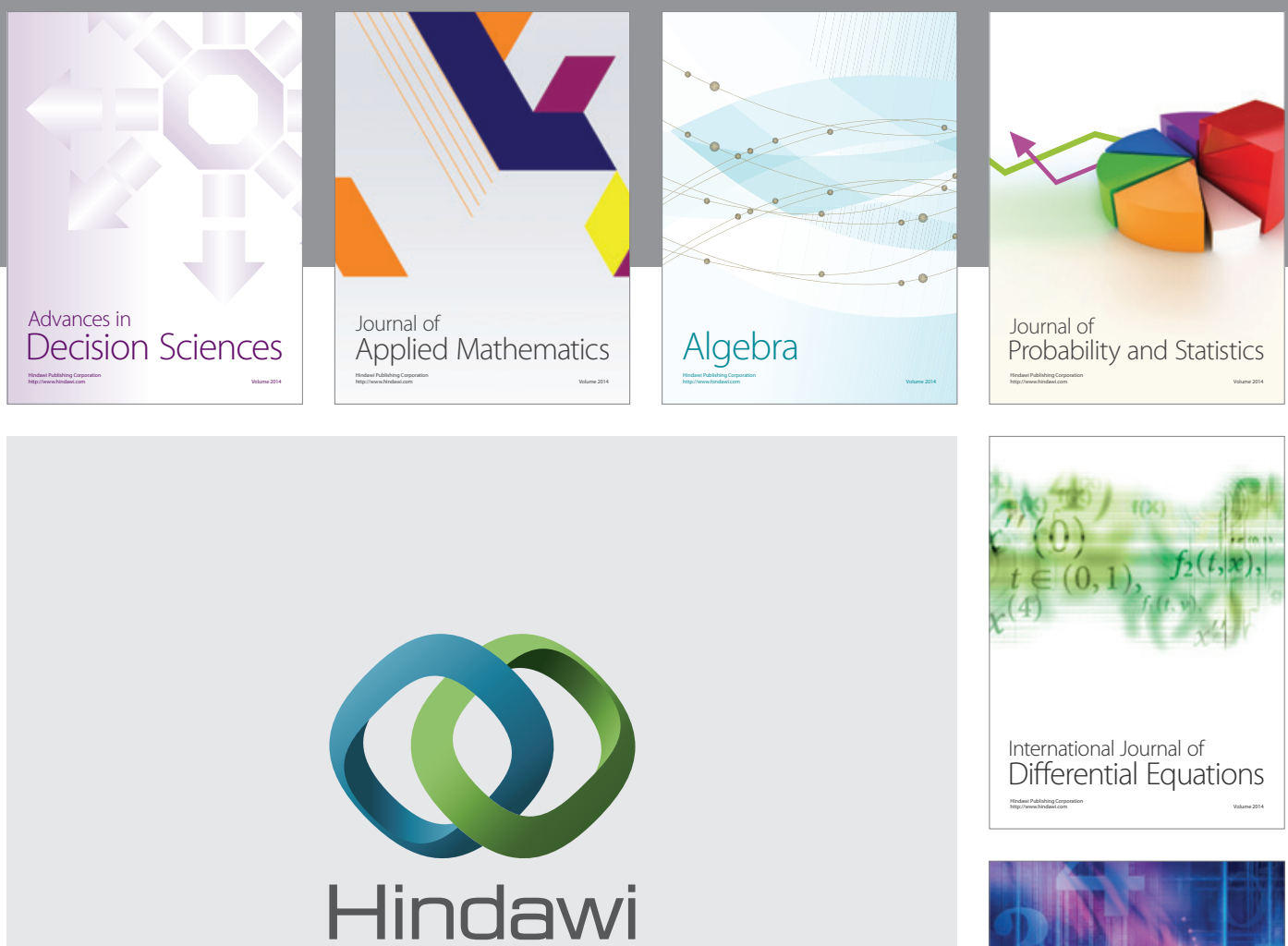

Submit your manuscripts at http://www.hindawi.com
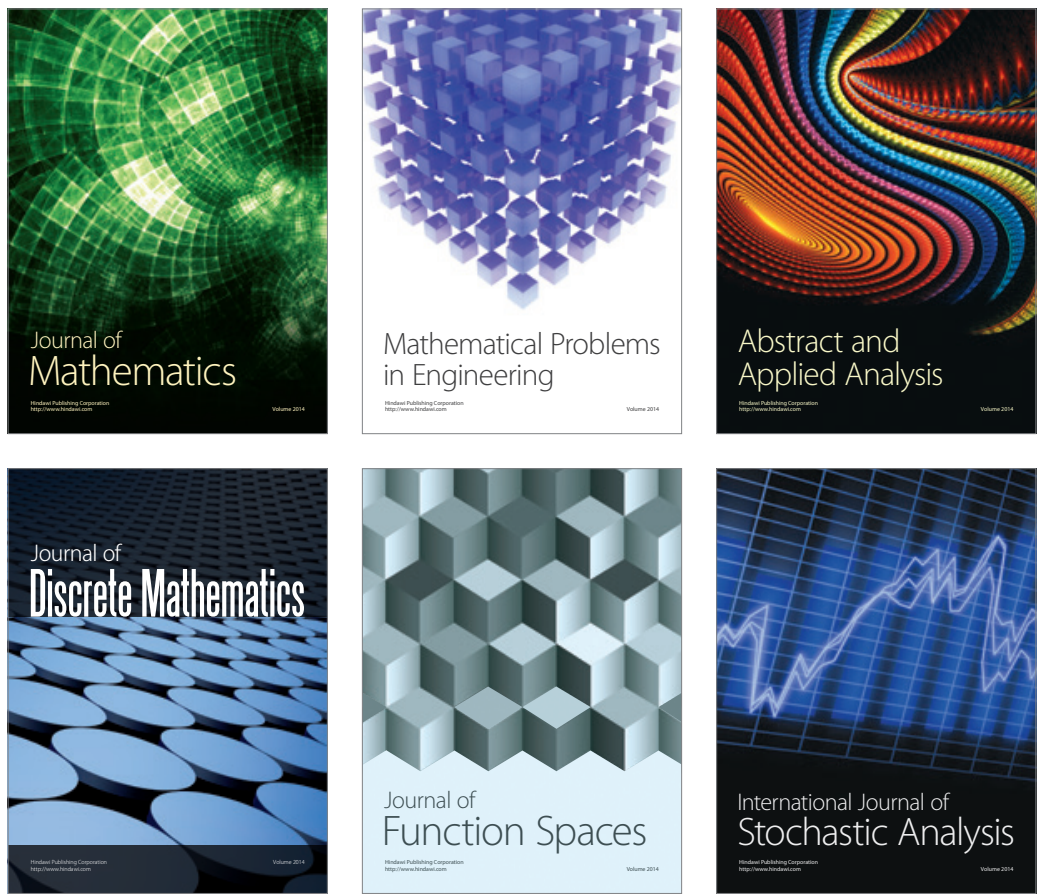

Journal of

Function Spaces

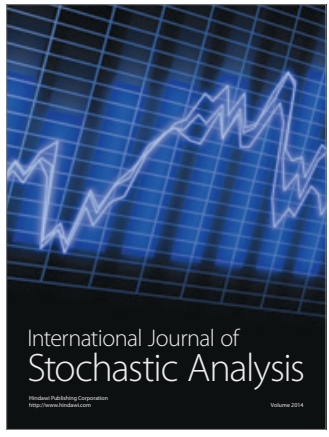

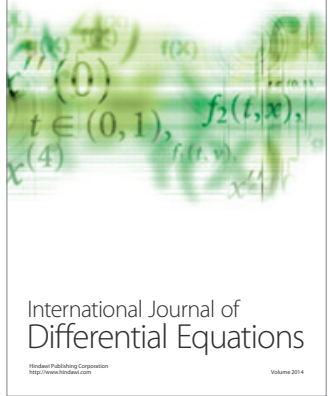
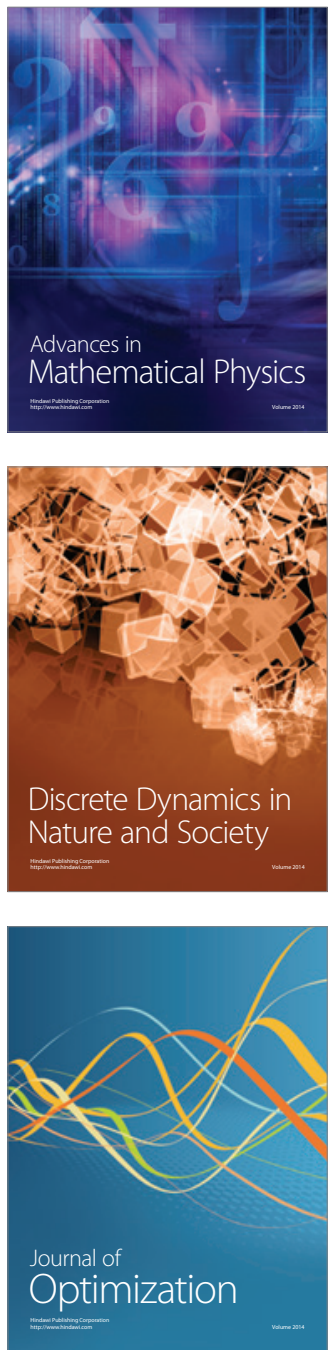